\title{
Inflammation as a potential mediator for the association between periodontal disease and Alzheimer's disease
}

\author{
Amber Watts' \\ Eileen M Crimmins' \\ Margaret Gatz ${ }^{2}$ \\ 'Davis School of Gerontology; \\ ${ }^{2}$ Department of Psychology, University \\ of Southern California, Los Angeles, \\ CA, USA
}

\begin{abstract}
Periodontal disease (PDD) is associated with increased risk of cardiovascular disease, cerebrovascular disease, and mortality in many studies, while other studies have begun to suggest an association of PDD with Alzheimer's disease (AD). This paper discusses how infectious pathogens and systemic infection may play a role in AD. The roles of infection and inflammation are addressed specifically with regard to known AD pathologic lesions including senile plaques, neuron death, neurofibrillary tangles, and cerebrovascular changes. A testable model of proposed pathways between periodontal infection and $\mathrm{AD}$ is presented including three possible mechanisms: a) direct effects of infectious pathogens, b) inflammatory response to pathogens, and c) the effects on vascular integrity. The role of gene polymorphisms is discussed, including apolipoprotein (APOE) $\varepsilon 4$ as a pro-inflammatory and pro-infection genotype.
\end{abstract}

Keywords: periodontal disease, infection, inflammation, Alzheimer's disease

Periodontal disease (PDD) is associated with increased risk of vascular disease and mortality in more than 200 reports and reviews (eg, DeStefano et al 1993; Beck et al 1996; Garcia et al 1998; Loesche et al 1998a, 1998b; Arbes et al 1999; Wu et al 2000; Grau et al 2004). Far fewer studies have examined the relationship between oral health earlier in life and Alzheimer's disease (AD) in late life (Kondo et al 1994; Gatz et al 2006; Kim et al 2007; Stein et al 2007). Inflammation is recognized as a core process in atherosclerosis and cardiovascular disease (CVD), and may also have a major role in AD. Chronic inflammation, as measured by blood inflammatory markers (Table 1), is associated with increased risk for cognitive decline (Weaver et al 2002; Yaffe et al 2003; Alley et al 2008) and dementia (Schmidt et al 2002; Engelhart et al 2004; Tan et al 2007). PDD is a common source of chronic systemic infection. This review suggests mechanisms by which periodontal infection may promote AD. The proposed model gives a rationale for further experimental and clinical studies.

Research has documented declines in self-care and oral hygiene that occur in people with dementia (eg, Chalmers and Pearson 2005; Meurman and Hämäläinen 2006). Our focus, however, is periodontal disease in early and midlife when it is clear that oral disease is an antecedent to dementia and when there would be no reason to suspect that dementia could have led to the oral disease.

\section{Periodontal disease}

PDD is a group of conditions that cause inflammation and destruction of the gums, alveolar bone, and other structures that support the teeth. The etiology is complex involving the presence of pathogenic bacteria found in dental plaque and individual variation in host immune response. PDD is a common source of chronic systemic 
Table I Inflammatory markers associated with cognitive decline and dementia in epidemiological studies

\begin{tabular}{|c|c|}
\hline Cytokines & Associated with \\
\hline Interleukin I (IL-I) & Dementia (Framingham Study') \\
\hline \multirow[t]{3}{*}{ Interleukin 6 (IL-6) } & Cognitive Decline (MacArthur Studies ${ }^{2}$; \\
\hline & Health ABC Study ${ }^{3}$ ) \\
\hline & Dementia (Rotterdam Study ${ }^{4}$ ) \\
\hline Interleukin I0 (IL-I0) & Cognitive Decline (Leiden $85+$ Study $^{5}$ ) \\
\hline Tumor necrosis factor & Cognitive Decline (Leiden $85+$ Study $^{5}$ ) \\
\hline alpha $(\mathrm{TNF}-\alpha)$ & Dementia (Framingham Study') \\
\hline \multicolumn{2}{|l|}{ Acute Phase Proteins } \\
\hline \multirow[t]{4}{*}{ C-reactive protein (CRP) } & Cognitive Decline (Health ABC Study3; \\
\hline & Greek Community $\left.{ }^{6}\right)$ \\
\hline & Dementia (Rotterdam Study4; \\
\hline & Honolulu-Asia Aging Study ${ }^{7}$ ) \\
\hline \multirow[t]{3}{*}{$\alpha-I$ antichymotrypsin (ACT) } & Cognitive Decline (Longitudinal Aging \\
\hline & Study Amsterdam ${ }^{8}$ ) \\
\hline & Dementia (Rotterdam Study ${ }^{4}$ ) \\
\hline \multicolumn{2}{|l|}{ Cell Adhesion Molecules } \\
\hline $\begin{array}{l}\text { Intercellular adhesion } \\
\text { molecule (ICAM-I) }\end{array}$ & Cognitive Decline (Greek Community ${ }^{6}$ ) \\
\hline $\begin{array}{l}\text { Vascular cell adhesion } \\
\text { molecule (VCAM-I) }\end{array}$ & Cognitive Decline (Greek Community ${ }^{6}$ ) \\
\hline
\end{tabular}

Notes: 'Tan et al 2007; ${ }^{2}$ Weaver et al $2002 ;{ }^{3}$ Yaffe et al $2003 ;{ }^{4}$ Engelhart et al 2004; ${ }^{5}$ van Exel et al 2003; ${ }^{6}$ Dimopoulos et al 2006; ${ }^{7}$ Schmidt et al 2002; ${ }^{8}$ Dik et al 2005.

infection in humans (Garcia et al 2000; Li et al 2000; Taylor et al 2006). The bacterial pathogens most strongly implicated in chronic periodontal disease are Porphyromonas gingivalis, Tannerella forsythensis, Treponema denticola, and Actinobacillus actinomycetemcomitans.

Elevated levels of interleukin 1 (IL-1) have been found in gingival crevicular fluid (GCF) of patients with experimentally induced gingivitis and active periodontal disease (Masada et al 1990; Kinane et al 1992). In patients with advanced periodontitis, substantial reduction of IL-1 levels occurred after treatment (Masada et al 1990). The simultaneous presence of $p$. gingivalis and $t$. forsythensis has been associated with increased GCF levels of inflammatory mediators and associated with more severe disease (Airila-Mansson et al 2006). In gingivitis, inflammatory mediators in the GCF do not penetrate deeply into tissues. Gingivitis advances to periodontitis when bacteria evade clearance by neutrophils and penetrate the deeper tissues (Offenbacher 1996).

Even low-grade infections in the oral cavity may be associated with moderate, sub-clinical systemic inflammatory response indicated by blood elevations of C-reactive protein (CRP) and interleukin 6 (IL-6) (D'Aiuto and Tonetti 2004; Taylor et al 2006). Severe PDD can induce chronic inflammation and immune reactions that result in loss of bone and soft tissue that supports teeth in the jaws. Systemic inflammatory markers are commonly elevated in individuals with PDD. In a recent review (Loos 2005), 8 studies showed that blood leukocytes and plasma levels of CRP were consistently higher in patients with periodontitis compared to healthy controls. In one study, carriers of common oral anaerobic bacteria had higher plasma levels of CRP (Bretz et al 2005). Furthermore, the severity of periodontal infection has been correlated with serum levels of inflammatory markers. For example, in the Atherosclerosis Risk in Communities study (ARIC), older adults with more extensive periodontal pockets had one-third higher plasma CRP than those with mild PDD (Slade et al 2003). Other inflammatory proteins such as IL-6 and tumor necrosis factor alpha (TNF- $\alpha$ ) have been found to be elevated in advanced PDD. For example, individuals with extensive PDD had 2- to 4-fold higher mean plasma levels of IL- 6 and TNF- $\alpha$ than those with mild or no disease (Bretz et al 2005).

The success of reducing CRP and other inflammatory markers with periodontal treatment has been mixed. Intervention studies of moderate to severe PDD use standard therapeutic scraping of dental calculus (scaling and root planing) alone or in combination with antiinflammatory or antiinfective drugs. Scaling and root planing alone reduced CRP levels in one study (D'Aiuto and Tonetti 2004) but did not reduce CRP, interleukins, or TNF- $\alpha$ in two other studies (Fokkema et al 2003; Ide et al 2003). Use of antiinflammatory drugs in combination with scaling and root planing reduced CRP and haptoglobin in one study (Ebersole et al 1997) and in another study antiinfective drugs in combination with scaling and root planing reduced CRP, especially among those with elevated baseline CRP (Mattila et al 2002). Extraction of all teeth in advanced PDD patients caused a reduction in blood CRP, fibrinogen, white cells, platelets, and plasminogen activator inhibitor-1 in another study (Taylor et al 2006). Though the most effective treatment for reduction of inflammation in PDD remains unclear, treatment of periodontal infection may help to reduce systemic inflammation.

PDD has been associated with increased risk of mortality, cardiovascular disease, and stroke in a large number of studies. For example, in the National Health and Nutrition Examination Study (NHANES), a large US national sample, adults with periodontitis had a $46 \%$ increased risk of independent all-cause mortality and a $25 \%$ increased risk of coronary heart disease (DeStefano et al 1993). Other studies have shown that individuals with markers of poor oral health 
are at 2 to 4 times greater risk for stroke (Beck et al 1996; Loesche et al 1998b; Wu et al 2000; Grau et al 2004).

Severity of PDD has shown a dose-response relationship with disease outcomes and mortality (Loesche et al 1998a; Arbes et al 1999). A longitudinal dental study of veterans found that individuals with the deepest probing pocket depths and the greatest degree of alveolar bone loss had the highest independent mortality risk (Garcia et al 1998). Though not all studies have supported PDD as a risk for CVD and cerebrovascular disease, most reports support a modest relationship (see Scannapieco et al 2003 for a systematic review).

PDD may also be associated with increased risk for AD. In Swedish twins, Gatz and colleagues (2006) found that those who had lost half or more of their teeth before age 35 had a 1.7-fold greater risk for $\mathrm{AD}$, after controlling for other factors. Within a twin pair, the demented twin was four times more likely to have had worse oral health before age 35 . Similarly, a case control study of AD in Japan found that loss of more than half of adult teeth by age 50-60 increased AD risk by 2.6-fold (Kondo et al 1994). In this study, PDD occurring 20-30 years prior to dementia onset was the most frequent cause of tooth loss. In a study of 144 nuns, having few or no teeth predicted a 4.3-fold higher risk of dementia, but only among those who did not carry the apolipoprotein (APOE) $\varepsilon 4$ allele (Stein et al 2007). The study included dental records that indicated whether teeth were lost due to PDD or other causes. In a Korean community population, persons with fewer teeth were at 1.6-fold greater risk of dementia in subsequent years if they did not have their missing teeth replaced by dentures (Kim et al 2007). Teeth are commonly lost as a result of periodontal infection and dental caries, both of which are caused by exposure to bacteria. Less commonly, teeth are lost due to trauma. Studies that measure markers of periodontal infection, not just tooth loss, will be important to establishing whether oral health may be related to AD.

\section{Systemic infection: From mouth to bloodstream}

The mouth is a primary channel by which external organisms enter the body. Transient bacteremia occurs after tooth brushing and flossing as well as after normal dental procedures (Loos 2005; Forner et al 2006). In individuals with good oral and immune health, the transient bacteremia has few consequences. It is suspected that individuals with periodontal infection have higher levels of pathogenic accumulation and may experience transient bacteremia multiple times per day (Li et al 2000; Loos 2005; Forner et al 2006). Gram-positive and Gramnegative oral bacteria contain several components within their membranes that can induce pro-inflammatory cytokines including IL-1, IL-6, and TNF- $\alpha$. Lipopolysaccharide (LPS) endotoxin is the most often studied of these bacterial components (Beck et al 1996; Wilson et al 1996).

The oral cavity has several barriers, physical, electrical, and chemical, that inhibit penetration by pathogens. First, the surface epithelium tissue layer provides a physical barrier composed of tight junctions and a chemical barrier containing peptide antibiotics called defensins. An electrical barrier affects the flow of electrons between the oral cavity and microbes that are introduced. The high reduction potential in the oral cavity increases oxidation of local bacteria. Another layer of protection is an immunological layer of antibodyforming cells. Finally, the reticuloendothelial system protects through phagocytosis to engulf and destroy bacteria upon entry into the blood (Weinberg et al 1998; Loesche and Lopatin 2000). If these barriers are compromised by PDD, trauma, or immune suppression, microbes can disseminate to cause acute or chronic infection ( $\mathrm{Li}$ et al 2000). Once in the bloodstream, these bacteria can induce acute phase response characterized by increased white blood cell counts and the release of inflammatory cytokines.

Supragingival plaque, above the gums, contains dense layers of Gram-positive bacteria (see Figure 1). If these bacteria penetrate the epithelium, they may survive the oral cavity barriers and enter the bloodstream (Loesche and Lopatin 2000). Though not specifically associated with PDD, the number of these bacteria increase in conditions of poor oral hygiene and may leak into the blood and result in the inflammation of distant systems.

Subgingival plaque, below the gums, contains primarily Gram-negative organisms in individuals with disease (Loesche and Lopatin 2000). These get trapped in the space between the tooth and the gum tissue and provoke local inflammatory responses giving rise to PDD. PDD is characterized by chronic Gram-negative infection in which bacteria may enter the bloodstream via the epithelium. Ulcerations in the gingival epithelium allow bacteria to spread from the pocket between the tooth and gum into the capillaries of the epithelium and thus into systemic circulation. Bacterial endotoxin (LPS contained in bacterial cell membranes) may enter the bloodstream. In PDD, the large surface area of the pockets allows a larger degree of LPS endotoxin to enter compared to the intact endothelial lining and more limited pockets in individuals without disease. LPS induces cytokine production and its entry may alter blood coagulation and promote atherosclerosis and thrombogenesis (Valtonen 1991; Stoll et al 2004). Cytokines produced locally, for 


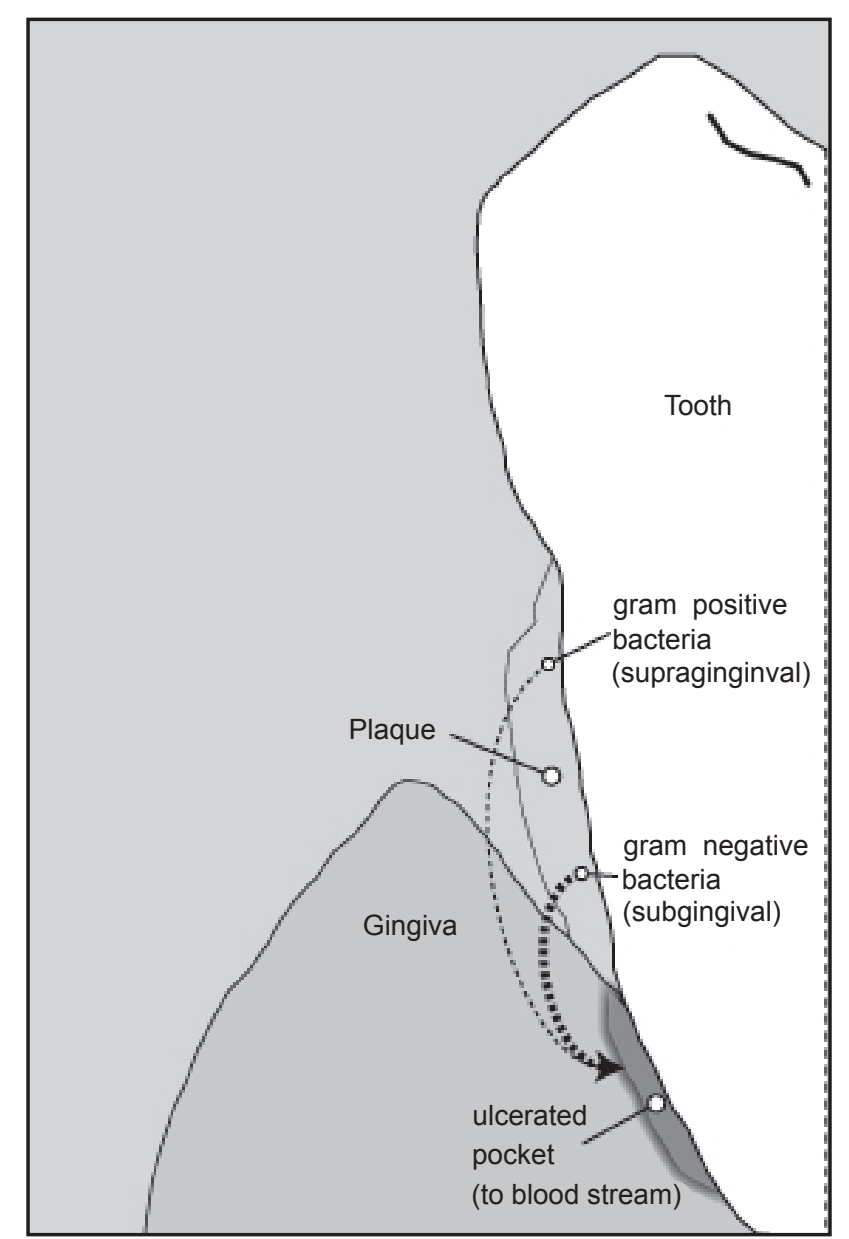

Figure I In periodontal disease, the gingiva recedes from the tooth forming pockets through which bacteria may more easily enter the bloodstream.

example in the periodontium, are generally degraded locally. However, under conditions of repeated challenge, cytokine receptors may become saturated and less able to eliminate cytokines, thus allowing them to "spill over" into systemic circulation affecting serum levels of cytokines and acute phase reactants (Offenbacher 1996). Chronic periodontal infection continuously attracts circulating leukocytes to inflamed periodontal tissues keeping immune cells activated (Fokkema et al 2003).

Bacteria are not the only pathogens that are likely to enter the system when defenses are vulnerable. Viruses are frequently detected in periodontal pockets (Kubar et al 2005). In one study of 30 patients with advanced periodontitis, $78 \%$ had at least one of five viruses and $40 \%$ were co-infected with two to five viruses (Parra and Slots 1996), particularly herpes viruses including cytomegalovirus (CMV), EpsteinBarr virus (EBV), and herpes simplex virus (HSV) found in $60 \%, 30 \%$, and $20 \%$ of patients, respectively. Herpes viruses also infect inflammatory cells in periodontitis
(Contreras et al 1999). The potential role of bacterial and viral pathogens in $\mathrm{AD}$ pathogenesis is discussed in more detail below.

\section{Transmigration from bloodstream to brain}

Though the blood-brain barrier (BBB) generally prevents entry of substances into the brain, there is evidence that inflammatory cytokines can enter or influence the brain under certain circumstances. For example, IL-6 and certain other blood cytokines elevated during the acute phase response enter the brain to initiate sickness behaviors including fever, malaise, reduced appetite, and decreased social contact (Perry 2004). These behaviors are a normal response that is adaptive to conserve energy stores when fighting infection and possibly protect others from the spread of disease. IL-6 and other cytokines may be transported across the intact BBB through specific transport processes (Pan and Kastin 1999; Banks et al 2002). IL-6 and other cytokines may also enter through fenestrated capillaries in circumventricular organs (including the pineal gland, vascular organ of the lamina terminalis, area postrem, and subfornical organ) near the base of the brain that are outside the $\mathrm{BBB}$ and are more easily penetrated (Banks et al 2002; Perry 2004).

\section{Infectious pathogens and AD}

Exposure to infectious pathogens of various types is a possible risk factor for $\mathrm{AD}$, although mixed results allow only tentative conclusions (Balin and Appelt 2001; Holmes et al 2003; Itzhaki et al 2004; Kinoshita 2004; Little et al 2004; Mattson 2004; Ringheim and Conant 2004; Robinson et al 2004; Wozniak et al 2005). Research has focused primarily on the bacterium Chlamydia pneumoniae, and herpes viruses including HSV and CMV. Very few studies have reported investigating the presence oral disease-related bacteria in AD (eg, Riviere et al 2002).

C. pneumoniae infection is characterized by chronic inflammation and is generally acquired orally or nasally. Although some studies found C. pneumoniae in post-mortem AD brains (Balin and Appelt 2001), other labs failed to replicate these results (Nochlin et al 1999; Gieffers et al 2000; Ring and Lyons 2000; Wozniak et al 2003). C. pneumoniae antigens have been located in microglia and astroglia cells in brain regions with $\mathrm{AD}$ neuropathology (Itzhaki et al 2004). An in vitro cell model of the BBB showed the possibility that $C$. pneumoniae could enter the brain (MacIntyre et al 2003). In other studies that have not been replicated, infective C. pneumoniae was obtained postmortem from an 
$\mathrm{AD}$ brain and induced amyloid plaques in a mouse model (Little et al 2004).

Treponema bacteria, a family of Gram-negative spirochetes commonly associated with PDD, was found in the brains of $\mathrm{AD}$ patients with greater frequency than in non- $\mathrm{AD}$ controls (Riviere et al 2002). The AD patients also had a greater variety of treponema species. The levels of bacteria measured in the blood did not differ between the $\mathrm{AD}$ patients and controls suggesting that $\mathrm{AD}$ subjects may be more susceptible to infection in the central nervous system (CNS) than controls. Antigens for two types of treponema were found in the trigeminal ganglia, pons, and hippocampus possibly indicating that the bacteria reached the brain via the trigeminal nerve. Other spirochetes have been found in the brain tissue, blood, and cerebrospinal fluid of $\mathrm{AD}$ patients (Miklossy 1993), however the finding was not confirmed in another study (McLaughlin et al 1999) and the link of spirochete presence to $\mathrm{AD}$ has been treated with skepticism (Hammond et al 1993).

Viral infection may also be a risk factor for $\mathrm{AD}$, particularly herpes viruses. Nondemented older adults with high serum levels of antibodies for CMV had faster rates of cognitive decline over a four year period than those with low levels of CMV antibodies (Aiello et al 2006). HSV-1 is found in a high proportion of non-AD elderly brains (Jamieson et al 1992) and is most often not found to be associated with dementia. This does not preclude the role of HSV in $\mathrm{AD}$, because presence of a virus is not sufficient to result in disease. Individual factors such as immune function, pathogen virulence, and genetic factors determine the outcome of exposure to a virus. Latent viruses may be reactivated by immunosuppression, stress, or inflammation in the brain (Itzhaki and Wozniak 2007). In AD brains, HSV-1 is present in areas of AD neuropathology. Remarkably, APOE $\varepsilon 4$ allele carriers showed more than 15 -fold risk of $\mathrm{AD}$ in the presence of HSV-1 in this sample. The APOE alleles may affect the degree or recurrence of viral damage, rather than susceptibility to infection with the virus (Itzhaki et al 1997; Itzhaki and Wozniak 2006). HSV-1 may contribute to the formation of amyloid plaques and abnormally phosphorylated tau protein, possibly by attenuating the processing of amyloid precursor protein (APP) into the toxic A $\beta$-peptide (Shipley et al 2005; Itzhaki and Wozniak 2007).

Infection with viruses early in life may put individuals at risk of becoming re-infected throughout life, even chronically (Robinson et al 2004). Since AD develops later in life, it is hypothesized that pathogens acquired early in life are not expressed until decades later. Age-related decline in immunity against pathogens or a long period of pathogen latency may offer explanations for the effects of pathogens found in older adulthood and rarely found in younger people (Itzhaki et al 2004; Robinson et al 2004). Systemic infection may result in the entry of cytokines into the brain and inflammation that reactivates latent HSV, further enhancing damage (Itzhaki and Wozniak 2006).

Viruses may infect multiple sites throughout the body including the periodontium and the brain without requiring a direct relationship between the different sites. It remains possible that PDD and AD are both partially, but independently, influenced by viruses.

\section{Inflammation and AD}

Inflammatory markers appear to be higher in persons with AD than normal age-matched controls. Blood IL-6 (Singh and Guthikonda 1997) and $\alpha 1$-antichymotrypsin (ACT) levels (Licastro et al 1995) are elevated in some AD patients compared to controls. In the brain, acute phase proteins IL-1, IL-6, S-100, CRP, and $\alpha 2$-macroglobulin are elevated in the temporal cortex of $\mathrm{AD}$ patients compared to controls (Griffin et al 1989; Wood et al 1993; McGeer et al 1994). CRP and serum amyloid $P$ are localized to the characteristic AD extracellular amyloid deposits and neuronal tau protein aggregates post-mortem (Kalaria 1992; Duong et al 1997). Microvessels from AD brains release $60 \%$ to $88 \%$ more IL-1 $\beta$, IL-6, and TNF- $\alpha$ than non-AD controls (Grammas and Ovase 2001). Though some AD patients have elevated systemic inflammatory markers, it is not clear whether systemic inflammation precedes dementia or if neuroinflammation itself might result in systemic inflammation. Longitudinal studies, discussed below, suggest that elevated systemic inflammation predicts dementia months to years later; however, neurological changes can precede clinical signs of dementia by more than a decade.

In community based samples, elevated blood inflammatory markers predict risk for dementia and incidence of cognitive impairment (Schmidt et al 2002; Engelhart et al 2004; Tan et al 2007; Alley et al 2008). In the Rotterdam Study, elevated blood IL-6, CRP, and ACT predicted increased risk of dementia onset over a year later (Engelhart et al 2004). Over a follow-up period of 25 years, men in the Honolulu-Asia Aging Study had a 3-fold increased risk for dementia in the top quartiles of inflammatory markers versus the lowest quartile at midlife (Schmidt et al 2002). In individuals with $A D$, elevated IL- $1 \beta$ predicted rates of cognitive decline (Holmes et al 2003). Patients with elevated markers preceding a baseline exam showed a greater rate of cognitive 
decline over a two-month follow-up period than those who did not have elevated levels prior to baseline. Though high levels of inflammatory markers predict dementia risk and cognitive decline in the demented, they may not be associated with decline in those with higher levels of normal cognitive function (Dik et al 2005; Alley et al 2008). For example, Dik and colleagues (2005) found that ACT was associated with declines in mental status, but not with measures of memory, fluid intelligence, or information processing speed.

\section{Model of proposed mechanisms for an association between periodontal infection and AD}

This model proposes possible links between oral infection and the pathology of AD. We do not claim that oral infection or inflammation are the causes of $\mathrm{AD}$, rather we propose that they may contribute to, exacerbate, and share risk factors with AD. Pathogenic bacteria in the oral cavity can lead to periodontal infection. Individuals vary in susceptibility to infection, partly due to the state of their oral hygiene and possibly due to particular genotypes that are more vulnerable to infection and have elevated inflammatory responses (discussed in more detail below). Once bacteria and/or viruses enter the blood stream, the infection may become systemic. From systemic circulation, pathogens and their products may cross the BBB and enter the brain. This may contribute to the development of AD pathology through three interrelated processes (see Figure 2). They are the direct effects of pathogenic products, the inflammatory response to these pathogens, and the effect on vascular integrity. These processes have been demonstrated to impact microglial activation, the production and formation of amyloid beta $(A \beta)$ and tau protein, and cerebrovascular pathology. Microglial activation is associated with neuron death in AD though the causal direction remains undetermined. $A \beta$ and tau proteins are central contributors to inter-related $\mathrm{AD}$ pathologies, plaques and neurofibrillary tangles.

\section{Pathogen products}

The cell walls of Gram-negative bacteria contain LPS that induces a number of host defenses. LPS stimulates certain inflammatory cytokines that are associated with microglial activation and altered processing of APP (Brugg et al 1995; Mattson 2004). Animal studies show that chronic infusion of LPS into rat brains may result in long lasting inflammatory reaction with pathological changes. These changes include an increased number of activated astrocytes, increased number and density of reactive microglia, increase in IL- $1 \beta$, TNF- $\alpha$, and beta amyloid precursor protein ( $\beta \mathrm{APP})$, the degeneration of hippocampal pyramidal neurons, impairment in spatial working memory, and decreased size of hippocampus and temporal lobe associated with increased lateral ventricles (Hauss-Wegrzyniak et al 2000).

Viruses could also contribute directly to AD pathology. HSV has glycoproteins that are very similar in amino acid sequences to $A \beta$ and tau protein and may aggregate like

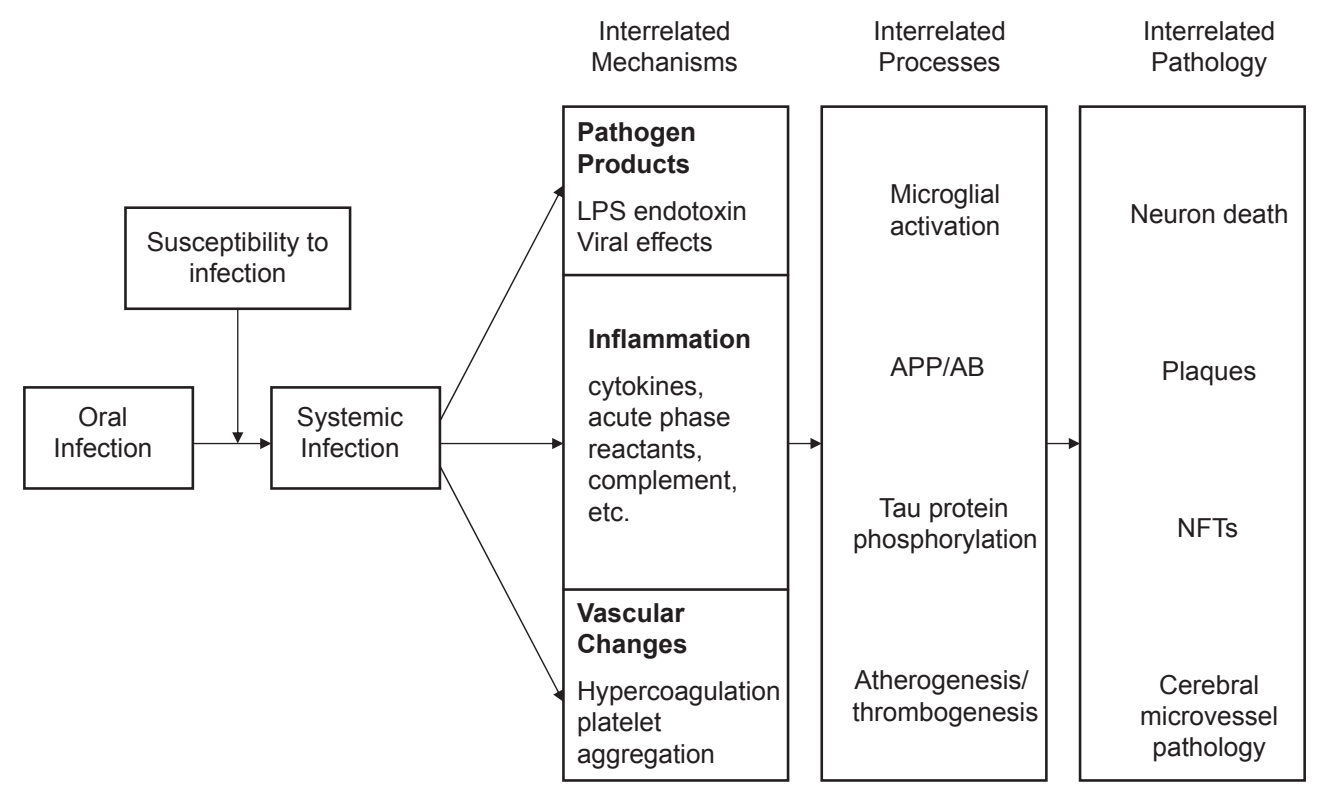

Figure 2 Proposed pathways between periodontal infection and Alzheimer's disease pathology. Abbreviations: LPS, lipopolysaccharide;APP, amyloid precursor protein;AB, amyloid beta; NFTs, neurofibrillary tangles. 
A $\beta$ (Cribbs et al 2000; Takakuwa et al 2001). HSV may also impact the processing of APP (Benboudjema et al 2003). For example, Shipley and colleagues (2005) reported that HSV-1 and HSV-2 infection of neuronal cells in vitro caused rapid decreases in cell levels of full length APP. The production of $A \beta$ may be increased as a result of altered APP processing or may also result directly from HSV infection (SatputeKrishnan et al 2003; Itzhaki and Wozniak 2006).

\section{Inflammation}

It is clear that inflammation is involved in AD. However, it is not yet clear if inflammatory processes initiate pathological processes or merely contribute to disease progression. There are several mechanisms through which systemic inflammation might contribute to pathogenesis in AD. These include the priming of microglia, dysregulation of APP and $\mathrm{A} \beta$ processing and metabolism, the activation of microglia in response to $A \beta$, and a neurotoxic loop or vicious cycle in which immune response intended to be neuroprotective leads to exacerbation of the process.

The presence of primed microglia may influence the response of the brain to systemic infection. In aging individuals, changes in glia can cause exaggerated inflammatory responses (Licastro et al 2005). It is hypothesized that in $\mathrm{AD}$ and other neurodegenerative diseases, microglia become activated, leading to higher production of inflammatory mediators and chronic overreaction to subsequent stimuli (Marx et al 1998; Perry 2004). Microglia release many inflammatory mediators in the brain including acute phase proteins, complement factors, prostaglandins, free radicals, and cytokines (Perry 2004). A $\beta$ peptides in the brain may also potentiate monocyte transmigration from blood to brain (Brod 2000).

The processing and metabolism of APP and $A \beta$ is critical in $\mathrm{AD}$ pathogenesis (Blasko and Grubeck-Loebenstein 2003; von Bernhardi et al 2007). In $\mathrm{AD}$, this balance is dysregulated. $A \beta$ is aggregated as oligomers and fibrils which have varying neurotoxicity (Klein et al 2001). Systemic inflammation and cytokine production can augment the regulation of APP and A $\beta$, though the effects are complex and may depend on which combination of mediators is present (Marx et al 1998; Blasko and Grubeck-Loebenstein 2003; Dziedzic 2006; Heneka and O'Banion 2007).

Griffin and colleagues (1998) proposed that IL-1 is critical to the processing of $\beta A P P$, favoring continued $A \beta$ deposition and the cyclical continuation of inflammatory response and cytokine overexpression. TNF- $\alpha$ and interferon gamma in combination also alter the metabolism of $\beta$ APP, trigger A $\beta$ peptide production, and inhibit soluble APP secretion
(Blasko et al 1999). IL-1 stimulates APP synthesis and factors that lead to its amyloidogenic properties (Schmitt et al 1996), contributes to the phosphorylation of tau protein favoring tangle formation, increases production of nitric oxide synthase fatal to cells, and increases the production of acetylcholinesterase responsible for the breakdown of acetylcholine which is important in learning and memory function (Mrak and Griffin 2005). Beta secretase, a protease that cleaves APP and results in toxic $A \beta$ peptides, may also be upregulated by inflammatory mediators (Heneka and O'Banion 2007). In mice with APP gene mutations, systemic administration of LPS resulted in altered expression and processing of APP and increased production of $A \beta$ (Sheng et al 2003). Mice with this gene mutation generate high levels of amyloidogenic $A \beta$.

$\mathrm{A} \beta$ and its aggregation trigger the activation of microglial cells which respond by producing acute-phase proteins, complement components, prostaglandins, and cytokines some of which are neurotoxic or contribute to aggregation by stimulating $A \beta$ production. This response may be more injurious than the plaques and tangles to which inflammatory processes are responding, resulting in neural damage and death (McGeer and McGeer 1995; Marx et al 1998; Neuroinflammation Working Group 2000; Wilson et al 2002; Blakso and Grubeck-Loebenstein 2003; Bate and Williams 2004; D’Andrea et al 2004; Nagele et al 2004; Dziedzic 2006; von Bernhardi et al 2007).

Nonspecific responses of phagocytic cells can remove $A \beta$ deposits, as well as recruit more microglia which produce more immune mediators. Cell attempts to dissolve plaques and tangles may cause toxicity via the release of neurotoxic substances including nitric oxide and other reactive oxygen species (Marx et al 1998; Heneka and O'Banion 2007). Proinflammatory cytokines including IL- 6 and TNF- $\alpha$ can be directly toxic in high concentrations or can stimulate further A $\beta$ production, aggregation, and toxicity (Marx et al 1998; Brod 2000; Blakso and Grubeck-Loebenstein 2003; Perry 2004; Heneka and O'Banion 2007). Chronic inflammation may result in chronic acute-phase protein secretion which favors the formation of $\mathrm{A} \beta$ fibrils. This fibrillar conformation of $A \beta$ may be important in its ability to induce inflammation (Blasko and Grubeck-Loebenstein 2003). Over sustained periods of time, these products may contribute to neurodegeneration via injury of surrounding noninfected cells resulting in neuron loss (Rogers et al 2002).

\section{Vascular changes}

Oral bacteria including Streptococcus sanguis and $P$. gingivalis have been shown to result in the expression 
of platelet aggregation proteins that may play a role in the formation of atheromas and thrombi possibly contributing to vascular disease (Herzberg and Meyer 1996, 1998; Sharma et al 2000; Pham et al 2002). High levels of atherosclerosis have been found to increase the risk of cognitive decline independent of other factors (Haan et al 1999). Furthermore, the presence of inflammatory cytokines in the blood increases platelet aggregation in cerebral blood vessels which can lead to atherogenesis and thrombus formation associated with strokes and hypoperfusion.

Vascular changes in the brain may also contribute to the formation of AD pathology. Platelets are a primary source of APP (Bush et al 1990; Chen et al 1995; Zandi and Breitner 2001) and platelet aggregation associated with cerebrovascular pathology may increase the production of $A \beta$ in the brain. In endothelial cells, $A \beta$ causes the secretion of inflammatory proteins which upregulate the production of APP (Grammas and Ovase 2001).

\section{Possible role of genes in infection and inflammation}

Acquisition of infectious disease requires the presence of both a pathogen and a susceptible host. The expression of disease depends on virulence of the pathogen and immune response of the host to the pathogen (Beck et al 2000). Periodontal researchers (Beck et al 1996) hypothesize a hyperinflammatory phenotype that causes some individuals to have exaggerated inflammatory reactions in response to pathogens. Particular alleles in the HLA-DR3/4 or -DQ system are suspected to produce exaggerated inflammatory responses to infection. Carriers have peripheral blood monocytes that secrete 3- to 10-fold greater amounts of inflammatory mediators in response to LPS. For example, patients with refractory periodontitis, a condition in which oral health does not improve despite treatment and proper hygiene, release higher levels of IL- $1 \beta$ and prostaglandin-E2 than patients with nonrefractory periodontitis (Hernichel-Gorbach et al 1994).

Polymorphisms for genes involved in inflammatory process are a logical target for exploration in understanding a possible common cause or link between periodontal disease and Alzheimer's disease. Several candidate genes have been proposed that may potentially link PDD and CVD (Kornman et al 1999; Kornman and Duff 2001; Goteiner et al 2008). While no publications have directly considered gene polymorphisms common to PDD and AD, IL-1 and TNF- $\alpha$ polymorphisms have been associated with both diseases (Diehl et al 1999; Galbraith et al 1999; Grimaldi et al 2000; Nicoll et al 2000; McCusker et al 2001; Raneiro et al 2004).
Not all studies have confirmed these associations (Craandijk et al 2002; Folwaczny et al 2004; Donati et al 2005) and more research on genetic polymorphisms is needed to explain discrepant findings (Loos et al 2005).

The APOE $\varepsilon 4$ allele is implicated in susceptibility to infection or the degree of resulting damage from viruses (Itzhaki et al 1997; Lin et al 1998, 2001; Wozniak et al 2005). AD brains positive for HSV were 17 times more likely to carry the APOE $\varepsilon 4$ allele compared to HSV-negative non$\mathrm{AD}$ brains suggesting that the combination of an APOE $\varepsilon 4$ allele and HSV may confer higher risk for AD than either alone (Itzhaki et al 1997).

APOE $\varepsilon 4$ alleles may also play a role in inflammatory response to infection. For example, mice with APOE $\varepsilon 4$ genotypes have greater elevations of inflammatory cytokines in systemic circulation and in the brain in response to LPS than those with APOE $\varepsilon 3$ (Lynch et al 2003). The ability of astroglial cells to phagocytize A $\beta$ may also depend on APOE type (Heneka and O'Banion 2007). ApoE and other genetic markers of hyperinflammatory response could be used to identify individuals at risk for targeted prevention and treatment.

\section{Establishing a relationship}

Many more studies are needed to validate an association between periodontal disease and dementia, including prospective studies that directly measure oral microbiology. Several requirements must be met before a causal relationship between PDD and AD can be established. First, PDD must precede dementia. Among the few longitudinal or archival studies that have reported tooth loss as a risk factor for dementia, two have measured periodontal disease as a possible cause for the tooth loss (Shimazaki et al 2001; Stein et al 2007), although none has established periodontal disease per se as the risk factor for dementia. Next, PDD must be correlated with AD. A few studies have suggested this is to be the case (eg, Kondo et al 1994; Gatz et al 2006; Stein et al 2007) although tooth loss was used as the index of PDD in these studies. Finally, all other possible contributors must be controlled for. While this is not entirely possible, steps have been and could be taken to account for some potential contributing factors. This includes external factors that might contribute to both tooth loss and dementia separately such as viral infection in different body systems, head injury, low socioeconomic status, malnutrition, or an exaggerated inflammatory profile. Eventually, randomized-controlled intervention trials with a long follow-up period would be needed to establish 
whether preventive oral health measures could reduce the risk of $\mathrm{AD}$.

For inflammation to be clearly established as a mediator for the relationship between PDD and dementia, it should be present in both oral disease and dementia as has been demonstrated through several studies (Licastro et al 1995; Singh and Guthikonda 1997; Loos 2005). Treating PDD should reduce inflammation (Ebersole et al 1997; Mattila et al 2002; D'Aiuto et al 2004; Taylor et al 2006) and reducing inflammation should lead to reduced incidence of dementia. Epidemiological studies suggest that nonsteroidal antiinflammatory drugs (NSAIDs) may protect against the development of $\mathrm{AD}$ if taken in midlife, many years prior to the diagnosis of dementia (eg, Hayden et al 2007). However, evidence from epidemiological studies, fundamental pathology, and clinical trials suggest that COX-2 inhibitors fail to reduce AD pathology and have not been shown to be effective in individuals who have already developed dementia (Townsend and Pratico 2005; McGeer and McGeer 2007).

No published studies have measured inflammation concurrently with PDD and dementia to allow a determination of its role in the relationship. It is necessary to determine whether inflammatory processes in the brain are initiated or exacerbated by systemic infection and inflammation resulting from PDD, or whether they reflect completely independent sources (eg, immune response to AD pathology).

\section{Conclusion}

This review and synthesis of the association between PDD and AD integrates research from disparate fields. Despite evidence that oral disease may be a risk factor for dementia, little attention has been devoted to explaining the potential mechanisms for this association (see Stein et al 2006). We propose that bacterial and viral infections commonly found in PDD may impact the brain, either directly or via systemic signals to the brain, and contribute to the development of AD. Periodontal infections may result in harmful pathogenic products leading to systemic inflammatory responses. Elevated systemic inflammatory response may contribute to the exacerbation of existing brain pathologies. Infections may also contribute to vascular pathology with the potential to impact brain function. PDD and AD may also share common risk factors such as genetic polymorphisms related to production of inflammatory mediators.

Though there are theoretical reasons for suspecting that pathogens may play a role in $\mathrm{AD}$, controversy remains given the mixed results of post-mortem brain studies
(Kinoshita 2004). Further evidence is needed to determine whether pathogens contribute uniquely to $\mathrm{AD}$ or are involved in a common cause. For example, perhaps those carrying the APOE $\varepsilon 4$ allele may have a hyperinflammatory response to challenges from pathogen products or be highly vulnerable to infection.

Few studies have attempted to link oral health with AD diagnosis or disease pathology and none have investigated the role of inflammation as a potential mediator. Information about history of chronic infection among AD patient populations would help to investigate this hypothesis. Longitudinal data measuring periodontal status, levels of inflammatory markers, and cognitive status would be ideal.

If systemic infection and inflammation prove to be contributors to AD, several preventive measures and treatment strategies would be implied. Such developments may be particularly significant given the paucity of promising preventative strategies for $\mathrm{AD}$ at the present time. Focus on the prevention of oral and other sources of systemic infection would be warranted. Timely treatment of periodontal infection might be indicated to reduce risk of systemic infection and inflammation. Though successful treatment of PDD has shown reductions in inflammation (eg, Taylor et al 2006), it remains to be determined whether this would reduce risk of CVD or dementia. Identification of individuals susceptible to infection and hyperinflammation would allow for targeted prevention to reduce contact with pathogens and treatment strategies to reduce harm from hyperinflammatory incidents.

The model presented here should enable researchers to test specific hypotheses regarding the multiple inter-related mechanisms that may be responsible for an association between oral infection, inflammation, and the development of Alzheimer pathology. In particular, research should examine whether pathogen products and inflammation resulting from periodontal infection are related to these same processes suspected to contribute to pathology in AD or whether the two diseases merely share common risk factors.

\section{Acknowledgments}

The authors acknowledge partial support from NIA Pilot Grant, P30 AG-017265, and NIA grant T32-AG00037, Center for Biodemography and Population Health University of Southern California/University of California Los Angeles. The authors would like to thank Caleb Finch and Jorgen Slots for helpful comments on earlier drafts of the paper and Bernard Steinman for his help in rendering Figure 1. 


\section{References}

Aiello AE, Haan MN, Blythe L, et al. 2006. The influence of latent viral infection on rate of cognitive decline over 4 years. J Am Geriatr Soc, 54:1046-54.

Airila-Mansson S, Soder B, Kari K, et al. 2006. Influence of combinations of bacteria on the levels of prostaglandin E2, interleukin-1 $\beta$, and granulocyte elastase in gingival crevicular fluid and on the severity of periodontal disease. J Periodontol, 77:1025-31.

Alley DE, Crimmins EM, Karlamangla A, et al. 2008. Inflammation and rate of cognitive change in high functioning older adults. J Gerontol A Biol Sci Med Sci, 63A:50-5.

Arbes SJ, Slade GD, Beck JD. 1999. Association between extent of periodontal attachment loss and self-reported history of heart-attack: An analysis of NHANES III data. J Dent Res, 78:1777-82.

Balin BJ, Appelt DM. 2001. Role of infection in Alzheimer's disease. J Am Osteopath Assoc, 101:S1-6.

Banks WA, Farr SA, Morley JE. 2002. Entry of blood-borne cytokines into the central nervous system: Effects on cognitive processes. Neuroimmunomodulation, 10:319-27.

Bate C, Williams A. 2004. Detoxified lipopolysaccharide reduces microglial cell killing of prion-infected neurons. NeuroReport, 15:2765-8.

Beck J, Garcia R, Heiss G, et al. 1996. Periodontal disease and cardiovascular disease. J Periodontol, 67:1123-37.

Beck JD, Slade G, Offenbacher S. 2000. Oral disease, cardiovascular disease, and systemic inflammation. Periodontology, 23:110-20.

Benboudjema L, Mulvey M, Gao Y, et al. 2003. Association of the herpes simplex virus type 1 US11 gene product with the cellular kinesin ligh-chain-related protein PAT1 results in the redistribution of both polypeptides. J Virol, 77:9192-203.

Blasko I, Grubeck-Loebenstein B. 2003. Role of the immune system in the pathogenesis, prevention and treatment of Alzheimer's disease. Drugs Aging, 20:101-13.

Blasko I, Marx F, Steiner E, et al. 1999. TNF $\alpha$ plus IFN $\gamma$ induce the production of Alzheimer $\beta$-amyloid peptides and decrease the secretion of APPs. FASEB J, 13:63-8.

Bretz WA, Weyant RJ, Corby PM, et al. 2005. Systemic inflammatory markers, periodontal disease, and periodontal infections in an elderly population. J Am Geriatr Soc, 53:1532-7.

Brod SA. 2000. Unregulated inflammation shortens human functional longevity. Inflamm Res, 49:561-70.

Brugg B, Dubreuil YL, Huber G, et al. 1995. Inflammatory processes induce $\beta$-amyloid precursor protein changes in mouse brain. Proc Natl Acad Sci US A, 92:3032-5.

Bush AI, Martins RN, Rumble B, et al. 1990. The amyloid precursor protein of Alzheimer's disease is released by human platelets. J Biol Chem, 265:15977-83.

Contreras A, Zadeh HH, Nowzari H, et al. 1999. Herpes virus infection of inflammatory cells in human periodontitis. Oral Microbiol Immunol, 14:206-12.

Chalmers J, Pearson A. 2005. Oral hygiene care for residents with dementia: A literature review. J Adv Nurs, 52:410-9.

Chen M, Inestrosa NC, Ross GS, et al. 1995. Platelets are the primary source of amyloid beta-peptide inhuman blood. Biochem Biophys Res Comm, 213:96-103.

Craandijk J, van Krugten MV, Verweij CL, et al. 2002. Tumor necrosis factor- $\alpha$ gene polymorphisms in relation to periodontitis. $J$ Clin Periodontol, 29:28-34.

Cribbs DH, Azizeh BY, Cotman CW, et al. 2000. Fibril formation and neurotoxicity by a herpes simplex virus glycoprotein B fragment with homology to the Alzheimer's A beta peptide. Biochemistry, 39:5988-94

D'Aiuto F, Tonetti D. 2004. Periodontal disease and C-reactive protein-associated cardiovascular risk. J Periodontal Res, 39:236-41.

D'Andrea MR, Cole GM, Ard MD. 2004. The microglial phagocytic role with specific plaques types in the Alzheimer disease brain. Neurobiol Aging, 25:675-83.
DeStefano F, Anda RF, Kahn HS, et al. 1993. Dental disease and risk of coronary heart disease and mortality. BMJ, 306:688-91.

Diehl SR, Wang Y, Brooks CN, et al. 1999. Linkage disequilibrium of interleukin-1 genetic polymorphisms with early-onset periodontitis. J Periodontol, 70:418-30.

Dik MG, Jonker C, Hack CE, et al. 2005. Serum inflammatory proteins and cognitive decline in older persons. Neurology, 64:1371-7.

Dimopoulos N, Piperi C, Salonicioti A, et al. 2006. Indices of low-grade chronic inflammation correlate with early cognitive deterioration in an elderly Greek population. Neurosci Lett, 398:118-23.

Donati M, Berglundh T, Hytonen AM, et al. 2005. Association of the -159 CD14 gene polymorphism and lack of association of the -308 TNFA and Q551R IL-4RA polymorphisms with severe chronic periodontitis in Swedish Caucasians. J Clin Periodontol, 32:474-9.

Duong T, Nikolaeva M, Acton PJ. 1997. C-reactive protein-like immunoreactivity in the neurofibrillary tangles of Alzheimer's disease. Brain Res, 749:152-6.

Dziedzic T. 2006. Systemic inflammatory markers and risk of dementia. Am J Alzheimers Dis Other Demen, 21:258-62.

Ebersole JL, Machen RL, Steffen MJ, et al. 1997. Systemic acute-phase reactants, C-reactive protein and haptoglobin in adult periodontitis. Clin Exp Immunol, 107:347-52.

Engelhart MJ, Geerlings MI, Meijer J, et al. 2004. Inflammatory proteins in plasma and the risk of dementia. Arch Neurol, 61:668-72.

Folwaczny M, Glas J, Torok HP, et al. 2004. Lack of association between the TNF- $\alpha$ G -308 A promoter polymorphism and periodontal disease. J Clin Periodontol, 31:449-53.

Fokkema SJ, Loos BG, deSlegte C, et al. 2003. Increased release of IL-12p70 by monocytes after periodontal therapy. J Clin Periodontol, 30:1091-6.

Forner L, Larsen T, Kilian M, et al. 2006. Incidence of bacteremia after chewing, tooth brushing, and scaling in individuals with periodontal inflammation. J Clin Periodontol, 33:401-7.

Galbraith GMP, Hendley TM, Sanders JJ, et al. 1999. Polymorphic cytokine genotypes as markers of disease severity in adult periodontitis, J Clin Periodontol, 26:705-9.

Garcia RI, Henshaw MM, Krall EA. 2000. Relationship between periodontal disease and systemic health. Periodontology, 25:21-36.

Garcia RI, Krall EA, Vokonas PS. 1998. Periodontal disease and mortality from all causes in the VA dental longitudinal study. Ann Periodontol, 3:339-49.

Gatz M, Mortimer JA, Fratiglioni L. 2006. Potentially modifiable risk factors for dementia in identical twins. Alzheimer Dement, 2:110-7.

Gieffers J, Reusche E, Solbach W, et al. 2000. Failure to detect Chlamydia pneumoniae in brain sections of Alzheimer's disease patients. J Clin Microbiol, 38:881-2.

Goteiner D, Ashmen R, Lehrman N, et al. 2008. Presence and significance of interleukin-1 polymorphism in patients who present with acute coronary syndrome, angina, and chronic periodontitis: An epidemiologic pilot study. J Periodontol, 79:138-43.

Grammas P, Ovase R. 2001. Inflammatory factors are elevated in brain microvessels in Alzheimer's disease. Neurobiol Aging, 22:837-42.

Grau AJ, Becher H, Ziegler CM, et al. 2004. Periodontal disease as a risk factor for ischemic stroke. Stroke, 35:496-501.

Griffin WST, Stanley LC, Ling C, et al. 1989. Brain interleukin 1 and S-100 immunoreactivity are elevated in Down syndrome and Alzheimer's disease. Proc Natl Acad Sci US A, 86:7611-5.

Griffin WST, Sheng JG, Royston MC, et al. 1998. Glial-neuronal interactions in Alzheimer's disease: The potential role of a 'cytokine cycle' in disease progression. Brain Pathol, 8:65-72.

Grimaldi LME, Casadei VM, Ferri C, et al. 2000. Association of early-onset Alzheimer's disease with an interleukin-1 $\alpha$ gene polymorphism. Ann Neurol, 47:361-5.

Haan MN, Shemanski L, Jagust WJ, et al. 1999. The role of APOE $\varepsilon 4$ in modulating effects of other risk factors for cognitive decline in elderly persons. JAMA, 281:40-6.

Hammond RR, Gage FH, Terry RD. 1993. Alzheimer's disease and spirochetes; a questionable relationship. Neuroreport, 4:840. 
Hauss-Wegrzyniak B, Vraniak PD, Wenk GL. 2000. LPS-induced neuroinflammatory effects do not recover with time. Neuroreport, 11:1759-63.

Hayden KM, Zandi PP, Khachaturian AS, et al. 2007. Does NSAID use modify cognitive trajectories in the elderly? The Cache County Study. Neurology, 69:275-82.

Heneka MT, O'Banion MK. 2007. Inflammatory processes in Alzheimer's disease. J Neuroimmunol, 184:69-91.

Hernichel-Gorbach E, Kornman KS, Holt SC, et al. 1994. Host responses in patients with generalized refractory periodontitis. J Periodontol, $65: 8-16$.

Herzberg MC, Meyer MW. 1996. Effects of oral flora on platelets: Possible consequences in cardiovascular disease. J Periodontol, 67:1138-42.

Herzberg MC, Meyer MW. 1998. Dental plaque, platelets, and cardiovascular diseases. Ann Periodontol, 3:151-60.

Holmes C, El-Okl M, Williams AL, et al. 2003. Systemic infection, interleukin 1 beta, and cognitive decline in Alzheimer's disease. $J$ Neurol Neurosurg Psychiatry, 74:788-9.

Ide M, McPartlin D, Coward PY, et al. 2003. Effect of treatment of chronic periodontitis on levels of serum markers of acute-phase inflammatory and vascular responses. J Clin Periodontol, 30:334-40.

Itzhaki RF, Wozniak MA. 2006. Herpes simplex virus type 1, apolipoprotein E, and cholesterol: A dangerous liaison in Alzheimer's disease and other disorders. Prog Lipid Res, 45:73-90.

Itzhaki RF, Wozniak MA. 2007. Viral infection and cognitive decline. $J$ Am Geriatr Soc, 55:131.

Itzhaki RF, Wozniak MA, Appelt DM, et al. 2004. Infiltration of the brain by pathogens causes Alzheimer's disease. Neurobiol Aging, 25:619-27.

Itzhaki RF, Lin W, Shang D, et al. 1997. Herpes simplex virus type 1 in the brain and risk of Alzheimer's disease. Lancet, 349:241-4.

Jamieson GA, Maitland MJ, Wilcock GK, et al. 1992. Herpes simplex virus type 1 DNA is present in specific regions of brain from aged people with and without senile dementia of the Alzheimer type. J Pathol, 167:365-8.

Kalaria RN. 1992. Serum amyloid P and related molecules associated with the acute-phase response in Alzheimer's disease. Res Immunol, 143:637-41.

Kim J, Stewart R, Prince M, et al. 2007. Dental health, nutritional status and recent-onset dementia in a Korean community population. Int $J$ Geriatr Psychiatry, 22:850-5.

Kinane DF, Winstanley FP, Adonogianaki E, et al. 1992. Bioassay of interleukin-1 (IL-1) in human gingival crevicular fluid during experimental gingivitis. Arch Oral Biol, 37:153-6.

Kinoshita J. 2004. Pathogens as a cause of Alzheimer's disease. Neurobiol Aging, 25:639-40.

Klein WL, Krafft GA, Finch CE. 2001. Targeting small A $\beta$ oligomers: The solution to an Alzheimer's disease conundrum? Trends Neurosci, 24:219-24.

Kondo K, Niino M, Shido K. 1994. A case-control study of Alzheimer's disease in Japan: Significance of lifestyles. Dementia, 5:314-26.

Kornman KS, Duff GW. 2001. Candidate genes as potential links between periodontal and cardiovascular disease. Ann Periodontol, 6:48-57.

Kornman KS, Pankow J, Offenbacher S, et al. 1999. Interleukin-1 genotypes and the association between periodontitis and cardiovascular disease. J Periodontal Res, 34:353-7.

Kubar A, Saygun I, Ozdemir A, et al. 2005. Real-time polymerase chain reaction quantification of human cytomegalovirus and Epstein-Barr virus in periodontal pockets and the adjacent gingiva of periodontitis lesions. J Periodontal Res, 40:97-104.

Li X, Kolltveit KM, Tronstad L, et al. 2000. Systemic diseases caused by oral infection. Clin Microbiol Rev, 13:547-58.

Licastro F, Candore G, Lio D, et al. 2005. Innate immunity and inflammation in ageing: A key for understanding age-related disease. Immun Ageing, 2:8-22.

Licastro F, Morini MC, Polazzi E, et al. 1995. Increased serum $\alpha 1$-antichymotrypsin in patients with probable Alzheimer's disease: An acute phase reactant without the peripheral acute phase response. J Neuroimmunol, 57:71-5.
Lin WR, Graham J, MacGowan SM, et al. 1998. Alzheimer's disease, herpes virus in brain, apolipoprotein E4 and herpes labialis. Alzheimers Rep, 1:173-8.

Lin WR, Wozniak MA, Esiri MM, et al. 2001. Herpes simplex encephalitis: involvement of apolipoprotein E genotype. J Neurol Neurosurg Psychiatry, 70:117-9.

Little CS, Hammond CJ, MacIntyre A, et al. 2004. Chlamydia pneumoniae induces Alzheimer-like amyloid plaques in brains of BALB/c mice. Neurobiol Aging, 25:419-29.

Loesche WJ, Schork A, Terpenning MS, et al. 1998a. Assessing the relationship between dental disease and coronary heart disease in elderly US veterans. J Am Dent Assoc, 129:301-11.

Loesche WJ, Schork A, Terpenning MS, et al. 1998b. The relationship between dental disease and cerebral vascular accident in elderly United States veterans. Ann Periodontol, 3:161-74.

Loesche WJ, Lopatin DE. 2000. Interactions between periodontal disease, medical diseases and immunity in the older individual. Periodontology, 16:80-105.

Loos BG. 2005. Systemic markers of inflammation in periodontitis. J Periodontol, 76:2106-15.

Loos BG, John RP, Laine ML. 2005. Identification of genetic risk factors for periodontitis and possible mechanisms of action. J Clin Periodontol, 32:159-79.

Lynch JR, Tang W, Wang H, et al. 2003. APOE genotype and an ApoE-mimetic peptide modify the systemic and central nervous system inflammatory response. $J$ Biol Chem, 278:48529-33.

MacIntyre A, Abramov R, Hammond CJ, et al. 2003. Chlamydia pneumoniae infection promotes the transmigration of monocytes through human brain endothelial cells. J Neurosci Res, 71:740-50.

Masada MP, Persson R, Kenney JS, et al. 1990. Measurement of interleukin$1 \alpha$ and $-1 \beta$ in gingival crevicular fluid: Implication for the pathogenesis of periodontal disease. J Periodontal Res, 25:156-63.

Mattila KJ, Vesanen M, Valtonen V, et al. 2002. Effect of treating periodontitis on C-reactive protein levels: A pilot study. BMC Infect Dis, $2: 30-2$.

Mattson MP. 2004. Infectious agents and age-related neurodegenerative disorders. Ageing Res Rev, 3:105-20.

Marx F, Blasko I, Pavelka M, et al. 1998. The possible role of the immune system in Alzheimer's disease. Exp Gerontol, 33:871-81.

McCusker, SM, Curran MD, Dynan KB et al. 2001. Association between polymorphism in regulation region of gene encoding tumour necrosis factor $\alpha$ and risk of Alzheimer's disease and vascular dementia: a casecontrol study. Lancet, 357:436-9.

McGeer PL, McGeer EG. 1995. The inflammatory response system of brain: Implications for therapy of Alzheimer and other neurodegenerative diseases. Brain Res Rev, 21:195-218.

McGeer PL, McGeer EG. 2007. NSAIDs and Alzheimer disease: Epidemiological, animal model and clinical studies. Neurobiol Aging, 28:639-47.

McGeer PL, Rogers J, McGeer EG. 1994. Neuroimmune mechanisms in Alzheimer disease pathogenesis. Alzheimer Dis Assoc Disord, 8:149-58.

McLaughlin R, Kin NM, Chen MF, et al. 1999. Alzheimer's disease may not be a spirochetosis. Neuroreport, 10:1489-91.

Meurman JH, Hämäläinen P. 2006. Oral health of people with dementia. Gerodontology, 23:3-32.

Miklossy J. 1993. Alzheimer's disease- a spirochetosis? Neuroreport, 4:841-8.

Mrak RE, Griffin WST. 2005. Glia and their cytokines in progression of neurodegeneration. Neurobiol Aging, 26:349-54.

Nagele RG, Wegiel J, Venkataraman V, et al. 2004. Contribution of glial cells to the development of amyloid plaques in Alzheimer's disease. Neurobiol Aging, 25:663-74.

Neuroinflammation Working Group. 2000. Inflammation and Alzheimer's disease. Neurobiol Aging, 21:383-421.

Nicoll JAR, Mrak RE, Graham DI, et al. 2000. Association of interleukin-1 gene polymorphisms with Alzheimer's disease. Ann Neurol, 47:365-8. 
Nochlin D, Shaw CM, Campbell LA, et al. 1999. Failure to detect Chlamydia pneumoniae in brain tissues of Alzheimer's disease. Neurology, $53: 1888$.

Offenbacher S. 1996. Periodontal disease: Pathogenesis. Ann Periodontol, 1:821-78.

Pan W, Kastin AJ. 1999. Penetration of neurotrophins and cytokines across the blood-brain/blood-spinal cord barrier. Adv Drug Deliv Rev, 36:291-8.

Parra B, Slots J. 1996. Detection of human viruses in periodontal pockets using polymerase chain reaction. Oral Microbiol Immunol, 11:289-93.

Perry VH. 2004. The influence of systemic inflammation on inflammation in the brain: implications for chronic neurodegenerative disease. Brain Behav Immuny, 18:407-13.

Pham K, Feik D, Hammond BF, et al. 2002. Aggregation of human platelets by gingipain- $\mathrm{R}$ from porphyromonas gingivalis cells and membrane vesicles. Platelets, 13:21-30.

Rainero I, Bo M, Ferrero M, et al. 2004. Association between the interleukin- $1 \alpha$ gene and Alzheimer's disease: a meta-analysis. Neurobiol Aging, 25:1293-8.

Ring RH, Lyons JM. 2000. Failure to detect Chlamydia pneumoniae in the late-onset Alzheimer's brain. J Clin Microbiol, 38:2591-4.

Ringheim, GE, Conant K. 2004. Neurodegenerative disease and the neuroimmune axis (Alzheimer's and Parkinson's disease, and viral infections). J Neuroimmunol, 147:43-9.

Riviere GR, Riviere KH, Smith KS. 2002. Molecular and immunological evidence of oral Treponema in the human brain and their association with Alzheimer's disease. Oral Microbiol Immunol, 17:113-8.

Robinson SR, Dobson C, Lyons J. 2004. Challenges and directions for the pathogen hypothesis of Alzheimer's disease. Neurobiol Aging, 25:629-37.

Rogers J, Strohmeyer R, Kovelowski CJ, et al. 2002. Microglia and inflammatory mechanisms in the clearance of amyloid beta peptide. Glia, 40:260-9.

Satpute-Krishnan P, DeGiorgia JA, Bearer EL. 2003. Fast anterograde transport of herpes simplex virus: role for the amyloid precursor protein of Alzheimer's disease. Aging Cell, 2:305-18.

Scannapieco FA, Bush RB, Paju S. 2003. Associations between periodontal disease and risk for atherosclerosis, cardiovascular disease, and stroke. A systematic review. Ann Periodontol, 8:38-53.

Schmidt R, Schmidt H, Curb JD, et al. 2002. Early inflammation and dementia: A 25-year follow-up of the Honolulu-Asia aging study. Ann Neurol, 52:168-74.

Schmitt T, Steiner E, Klingler P, et al. 1996. The production of an amyloidogenic metabolite of the Alzheimer amyloid $\beta$ precursor protein (APP) in thyroid cells is stimulated by interleukin $1 \beta$, but inhibited by interferon gamma. $J$ Clin Endocrinol Metabol, 81:1666-9.

Sharma A, Novak EK, Sojar HT, et al. 2000. Porphyromonas gingivalis platelet aggregation activity: Outer membrane vesicles are potent activators of murine platelets. Oral Microbiol Immunol, 15:393-6.

Sheng JG, Bora SH, Xu G, et al. 2003. Lipopolysaccharide-inducedneuroinflammation increases intracellular accumulation of amyloid precursor protein and amyloid $\beta$ peptide in APPswe transgenic mice. Neurobiol Dis, 14:133-45.

Shimazaki Y, Soh I, Saito T, et al. 2001. Influence of dentition status on physical disability, mental impairment, and mortality in institutionalized elderly people. $J$ Dent Res, 80:340-5.

Shipley SJ, Parkin ET, Itzhaki RF, et al. 2005. Herpes simplex virus interferes with amyloid precursor protein processing. BMC Microbiol, 5:48-55.

Singh VK, Guthikonda P. 1997. Circulating cytokines in Alzheimer's disease. J Psychiatr Res, 31:657-60.
Slade GD, Ghezzi EM, Heiss G, et al. 2003. Relationship between periodontal disease and C-reactive protein among adults in the Atherosclerosis Risk in Communities Study. Arch Intern Med, 163:1172-9.

Stein PS, Desrosier M, Donegan SJ, et al. 2007. Tooth loss, dementia and neuropathology in the Nun Study. J Am Dent Assoc, 138:1314-22.

Stein PS, Scheff S, Dawson DR. 2006. Alzheimer's disease and periodontal disease: Mechanisms underlying a potential bi-directional relationship. Grand Rounds in Oral Systemic Medicine, 3:14-24.

Stoll LL, Denning GM, Weintraub NL. 2004. Potential role of endotoxin as a proinflammatory mediator of atherosclerosis. Arterioscler Thromb Vasc Biol, 24:2227-36.

Takakuwa H, Goshima F, Koshizuka T, et al. 2001. Herpes simplex virus encodes a virion-associated protein which promotes long cellular processes in over-expressing cells. Genes Cells, 6:955-6.

Tan ZS, Beiser AS, Vasan RS, et al. 2007. Inflammatory markers and the risk of Alzheimer disease: The Framingham study. Neurology, 68:1902-8

Taylor BA, Tofler GH, Carey HMR, et al. 2006. Full mouth tooth extraction lowers systemic inflammatory and thrombotic markers of cardiovascular risk. J Dent Res, 85:74-8.

Townsend KP, Pratico D. 2005. Novel therapeutic opportunities for Alzheimer's disease: Focus on nonsteroidal anti-inflammatory drugs. FASEB J, 19:1592-601.

Valtonen VV. 1991. Infection as a risk factor for infarction and atherosclerosis. Ann Med, 23:539-43.

van Exel E, Craen AJM, Remarque EJ, et al. 2003. Interaction of atherosclerosis and inflammation in elderly subjects with poor cognitive function. Neurology, 61:1695-701.

von Bernhardi R, Ramirez G, Toro R, et al. 2007. Pro-inflammatory conditions promote neuronal damage mediated by amyloid precursor protein and decrease its phagocytosis and degradation by microglial cells in culture. Neurobiol Dis, 26:153-64.

Weaver JD, Huang MH, Albert M, et al. 2002. Interleukin-6 and risk of cognitive decline: MacArthur Studies of Successful Aging. Neurology, 59:371-8.

Weinberg A, Krisanaprakornkit S, Dale BA. 1998. Epithelial antimicrobial peptides: Review and significance for oral applications. Crit Rev Oral Biol Med, 9:399-414.

Wilson CJ, Finch CE, Cohen HJ. 2002. Cytokines and cognition: The case for a head-to-toe inflammatory paradigm. $J$ Am Geriatr Soc, 50:2041-56.

Wilson M, Reddi K, Henderson B. 1996. Cytokine-inducing components of periodontopathogenic bacteria. J Periodontal Res, 31:393-407.

Wood JA, Wood PL, Ryan R, et al. 1993. Cytokine indices in Alzheimer's temporal cortex: No changes in mature IL-1 $\beta$ or IL-1RA but increases in the associated acute phase proteins IL-6, $\alpha 2$ macroglobulin, and C-reactive protein. Brain Res, 629:245-52.

Wozniak MA, Cookson A, Wilcock GK, et al. 2003. Absence of chlamydia pneumoniae in brain of vascular dementia patients. Neurobiol Aging, 24:761-5.

Wozniak MA, Shipley SJ, Combrinck M, et al. 2005. Productive herpes simplex virus in brain of elderly normal subjects and Alzheimer's disease patients. J Med Virol, 75:300-6.

Wu T, Trevisan M, Genco RJ, et al. 2000. Periodontal disease and risk of cerebrovascular disease: The first National Health and Nutrition Examination Survey and its follow-up study. Arch Intern Med, 160:2749-55.

Yaffe K, Lindquist K, Penninx BW, et al. 2003. Inflammatory markers and cognition in well-functioning African-American and white elders. Neurology, 61:76-80.

Zandi PP, Breitner JCS. 2001. Do NSAIDs prevent Alzheimer's disease? And if so, why? The epidemiological evidence. Neurobiol Aging, 22:811-7. 